\title{
Tumor-specific delivery of KRAS siRNA with iRGD-exosomes efficiently inhibits tumor growth
}

\author{
Yu Zhou ${ }^{\dagger}$, Yuan Yuan ${ }^{\dagger}$, Minghui Liu, Xiuting Hu, Yiming Quan and Xi Chen
}

\begin{abstract}
Lung cancer is the major cause of cancer-related deaths globally. Mutant KRAS is a feature of $15-50 \%$ of lung cancer cases and represents one of the most prevalent oncogenic drivers in this disease. Unfortunately, although much effort has been spent on searching for small molecule inhibitors of KRAS, KRAS gene has proven extraordinarily difficult to target by current pharmacological agents. In the present study, we developed an alternative strategy to silence the so-called untargetable and undruggable KRAS gene by employing exosomemediated siRNA delivery. Particularly, we reprogrammed HEK293T cells to simultaneously express KRAS siRNA and Lamp2b, an exosomal membrane protein, in fusion with a tumor-penetrating internalizing RGD (iRGD) peptide (CRGDKGPDC), and then purified the tumor-targeting exosomes as KRAS siRNA delivery system. In agreement with the study design, intravenously injected iRGD-exosomes specifically targeted to tumor tissues in vivo. The therapeutic potential was revealed by the strong inhibition of tumor growth in a mouse model after intravenous injection of KRAS siRNA encapsulated in iRGD-exosomes. In conclusion, our results indicate that iRGD-tagged exosomes is an ideal delivery agent to transport KRAS siRNAs for lung cancer treatment. As an extension of this finding, the vast majority of mutated genes that are difficult to target by current pharmacological agents will be targetable and druggable in the future.
\end{abstract}

Keywords: Lung cancer, siRNA, KRAS, Exosome, iRGD

\section{Background}

Globally, lung cancer is diagnosed at the highest frequency and leads to the highest mortality amongst all cancers [1]. Lung cancers are often identified in patients at late phase due to its asymptomaticity which renders the current treatments unsensitive (e.g., surgery, radiotherapy and chemotherapy). Thus, designing new therapeutic agents for lung cancer is still crucial. The discovery of key signaling pathways involved in mediating cancer cell development and metastasis significantly expands our understanding of lung cancer biology. For example, oncogenic mutations such as EGFR and EML4-ALK have been intensively investigated as targets of personalized lung cancer therapy.

\footnotetext{
* Correspondence: xichen@nju.edu.cn

${ }^{\dagger}$ Yu Zhou and Yuan Yuan contributed equally to this work.

State Key Laboratory of Pharmaceutical Biotechnology, NJU Advanced Institute for Life Sciences, Jiangsu Engineering Research Center for MicroRNA Biology and Biotechnology, School of Life Sciences, Nanjing University, 163 Xianlin Avenue, Nanjing 210023, China
}

One of the commonest mutant genes in lung cancer cells is the Kirsten Rat Sarcoma viral oncogene homolog (KRAS) [2]. KRAS gene is located at 12p12.1 and encodes a protein that is a member of the small GTPase superfamily [3, 4]. KRAS is a prominent oncogene because it can transform normal cells into malignant cells, particularly when harboring an activating mutation in codon 12 or 13 [5]. Abnormal overexpression and somatic activating mutations in KRAS are universal in many cancer types, including majority of the pancreatic cancer (70-90\%) [6], 30-60\% of colon cancer [7] and 15-50\% of lung cancer patients [8]. Thus, the search for effective inhibitors for KRAS is an urgent need. Unfortunately, although much effort has been spent, KRAS has remained an untargetable and undruggable oncogene, possibly due to the absence of allosteric binding sites or activation of parallel signaling pathways that provide compensatory signals.

(c) The Author(s). 2019 Open Access This article is distributed under the terms of the Creative Commons Attribution 4.0 International License (http://creativecommons.org/licenses/by/4.0/), which permits unrestricted use, distribution, and reproduction in any medium, provided you give appropriate credit to the original author(s) and the source, provide a link to the Creative Commons license, and indicate if changes were made. The Creative Commons Public Domain Dedication waiver (http://creativecommons.org/publicdomain/zero/1.0/) applies to the data made available in this article, unless otherwise stated. 
Short interfering RNA (siRNA) has the potential to silence the so-called untargetable and undruggable genes based on recognition and subsequent degradation of specific mRNA sequences [9]. However, the successful implementation of siRNA in vivo is hampered by some major barriers: 1) Being target-specific based either on tissue or cell types; 2) the toxicity and immunogenicity of either siRNA or its delivery vehicle; 3 ) the instability of siRNA in circulation and difficulty to transfer across biological barriers. Thus, developing a new and efficient siRNA delivery system is quite essential.

Exosomes are endocytosis-relevant vesicles sized at 30$100 \mathrm{~nm}$ [10-12]. They are involved in intercellular interaction through mediating horizontal transfer of informative proteins, lipids and RNAs [13, 14]. Because exosomes can act as natural carriers of RNAs, they might provide a novel delivery strategy for siRNA [15]. The concept of using exosome for siRNA delivery was first proved by Alvarez-Erviti et al., who developed modified brain-specific exosome that expresses a brain-targeting peptide derived from RVG (rabies virus glycoprotein, YTIWMPENPRPGTPCDIFTNSRGKRASNG) fused to the N-terminus of Lamp2b (lysosome-associated membrane glycoprotein $2 \mathrm{~b}$ ), a protein that is abundant in exosome outer membrane. In principle, Lamp2b can guide the RVG peptide to exosomal surface, and the modified exosomes can recognize and target neuronal cells by binding to acetylcholine receptors on the blood-brain barrier and neuronal cells $[16,17]$. Intravenous injection such modified exosomes into normal mice led to efficient delivery of siRNA to the brain and consequently knockdown of BACE1 gene, highlighting the therapeutic value of this new RNAi strategy [18].

In this study, we developed a novel strategy for lung cancer treatment by employing exosome-mediated siRNA delivery. Tumor targeting was achieved by engineering HEK293T (human embryonic kidney $293 \mathrm{~T}$ ) cells to express Lamp2b in fusion with the tumor-penetrating iRGD peptide (CRGDKGPDC) which has a high affinity to integrin $\alpha v \beta 3$. Intravenously injected iRGD-exosomes delivered KRAS siRNA specifically to tumors in vivo, resulting in specific KRAS gene knockdown and tumor growth inhibition.

\section{Results}

\section{Characterization of the redesigned iRGD-exosomes loaded with KRAS siRNA}

We firstly designed a siRNA sequence targeting to the coding sequence homologue in both mouse and human KRAS. Then a plasmid that simultaneously expressed KRAS siRNA and the Lamp2b protein in fusion with an iRGD peptide (referred to here as siRNA-KRAS/iRGD) was constructed (Additional file 1: Figure S1). Theoretically, after collecting exosomes from HEK293T cells that express siRNA-KRAS/iRGD plasmid and injecting the redesigned exosomes into tumor-bearing mice, the iRGD tag that was engineered to be expressed on the exosomal membrane would guide the exosomes to tumor cells to release KRAS siRNA. Then the homogeneity of the siRNA-KRAS/iRGD-exosomes was confirmed by nanoparticle tracking analysis (NTA). The modified exosomes were physically homogenous and nano-sized, with a mode particle size of approximately $182 \mathrm{~nm}$ in diameter (Fig. 1a). The results suggest that the exosomal characteristics were not affected by our modifications (integration of KRAS siRNA and iRGD-Lamp2b fusion protein).

\section{KRAS siRNA encapsulated in iRGD-exosomes suppressed the proliferation of lung cancer cells}

In order to investigate whether or not the iRGD-exosomes could deliver KRAS siRNA to recipient tumor cells and induce KRAS downregulation, KRAS siRNA encapsulated in iRGD-tagged HEK293T exosomes was added to the cell culture medium of human lung carcinoma A549 cells. Wild type HEK293T exosomes (Mock) and scrambled RNA encapsulated in wild type HEK293T exosomes (referred to here as scrRNA/WT-exosome) served as controls. Then the ability of these exosomal RNA to knock down KRAS expression was assessed. After $6 \mathrm{~h}$, KRAS siRNA delivered by iRGD-tagged HEK293T exosomes reduced KRAS protein and mRNA levels in A549 cells (Fig. 1b and c), suggesting a high efficiency of siRNA-KRAS/iRGD-exosomes for KRAS interference. Next, the above experiment was repeated and the proliferation of A549 cells was assessed. The KRAS siRNA encapsulated in iRGD-tagged HEK293T exosomes showed an anti-proliferation effect on A549 cells, whereas natural HEK293T exosomes and scrambled RNA encapsulated in wild type HEK293T exosomes were not capable of inhibiting the growth of A549 cells (Fig. 2). Thus, KRAS siRNA encapsulated in iRGD-exosomes can inhibit tumor development in vitro.

\section{iRGD-exosomes specially targeted tumor tissues in vivo}

Next, we investigated whether iRGD-exosomes would be able to specifically deliver their cargo siRNA to lung cancer cells after systemic delivery. After the development of a tumor xenograft mouse model, scrRNA/ WT-exosomes and siRNA-KRAS/iRGD-exosomes were labeled with Dil dye (red fluorescence) and systemically injected into tumor-bearing mice through the tail vein. Then the bio-distribution of fluorescence-labelled exosomes was measured in mouse tumor, liver and kidney by fluorescence microscopy. Significant increased fluorescence signals were observed in tumors of mice treated with iRGD-tagged exosomes compared to those treated with unmodified exosomes (Fig. 3), suggesting that the iRGD peptide on exosomal surface specifically guides exosomes to tumor cells. As a control, iRGD tag did not affect fluorescence signal accumulation in liver and 
a

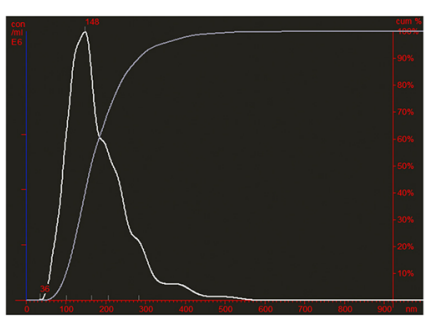

b

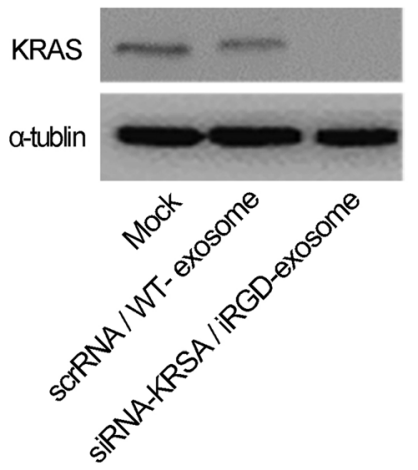

NanoSight

Size distribution (nm)

Mean:182, Mode:148, SD:77

Cumulative data (nm)

D10:101, D50:154, D90:283

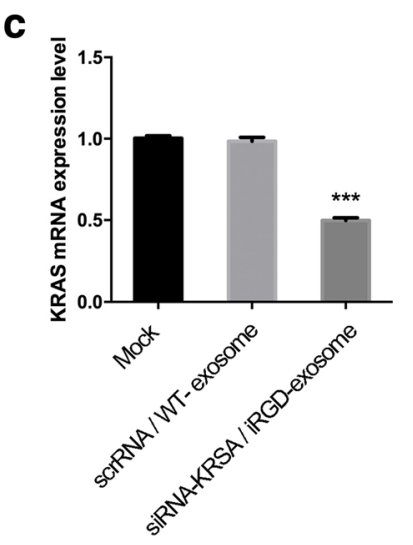

Fig. 1 Characterization of the iRGD-exosomes loaded with KRAS siRNA. (a) Representative images of nanoparticles tracking analysis (NTA) indicating similar size distribution in diluted samples of HEK293T cells exosomes. (b) Representative western blot image of KRAS protein in A549 cells treated with wild type HEK293T exosomes (Mock), scrRNA-loading wild type exosomes (scrRNAMT-exosome) or KRAS siRNA-loading iRGDexosomes (siRNA-KRAS/iRGD-exosome). (c) Quantitative RT-PCR analysis of KRAS mRNA levels in A549 cells treated with mock exosomes, scrRNA/ WT-exosomes or siRNA-KRAS/iRGD-exosomes. ${ }^{* * *}, p<0.001$

kidney (Fig. 3). Based on the data above, targeted delivery of siRNA with iRGD-tagged exosomes would result in efficient delivery of siRNA to the tumors.

KRAS siRNA encapsulated in iRGD-exosomes inhibited tumor development in a xenograft mouse model To evaluate the in vivo therapeutic potential of our tumor-specific siRNA delivery system, bioluminescent
A549 cells were subcutaneously implanted into nude mice to generate a xenograft tumor model. The tumors were left growing for 28 days post-implantation, and then the mice were intravenously injected with scrRNA/ WT-exosomes or siRNA-KRAS/iRGD-exosomes. After three times of injection of exosomes, tumor-bearing mice were scanned using non-invasive bioluminescent imaging (BLI) to evaluate tumor growth. While a strong

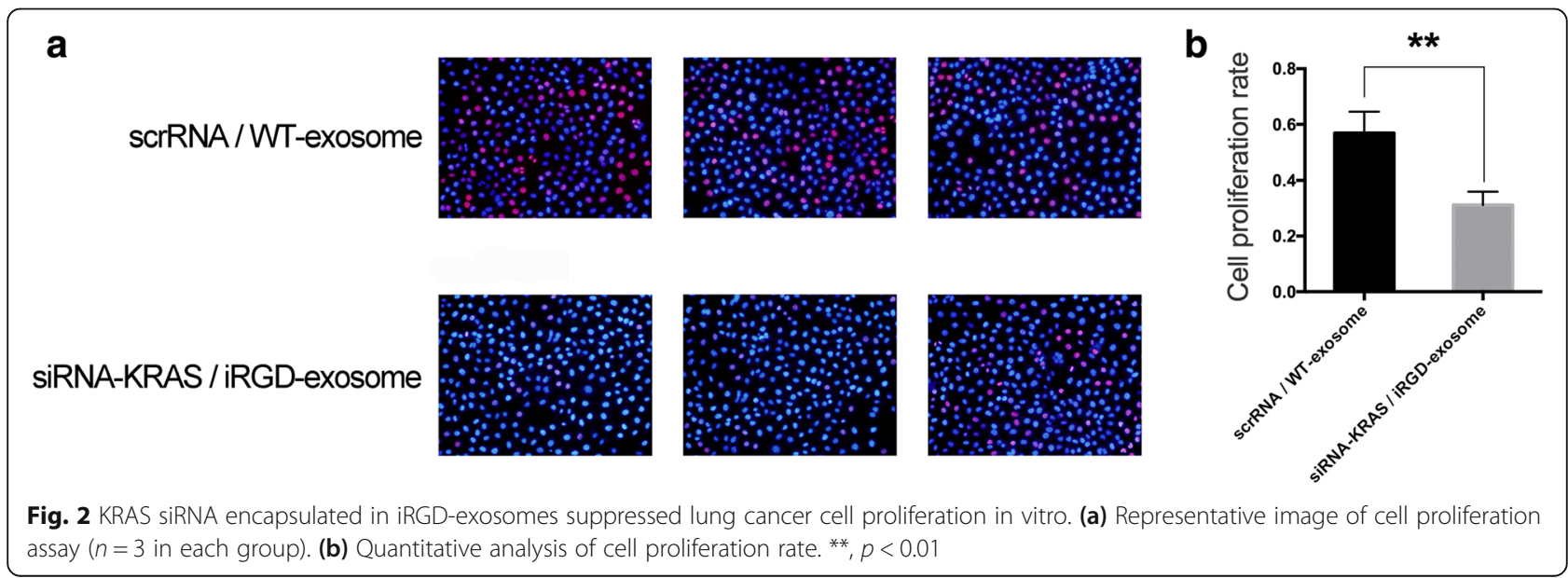


a

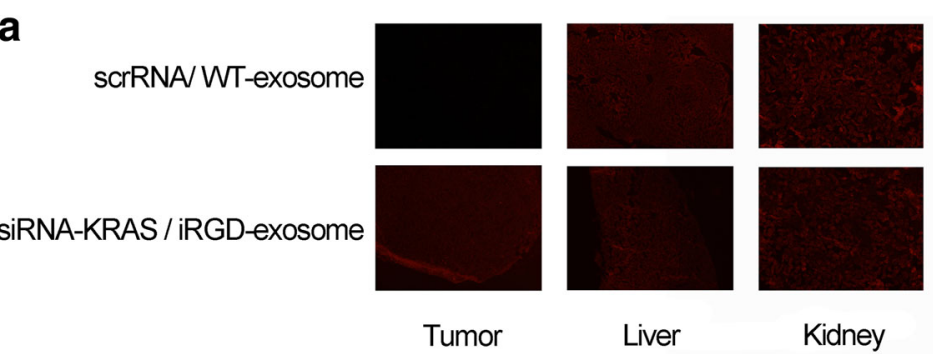

b

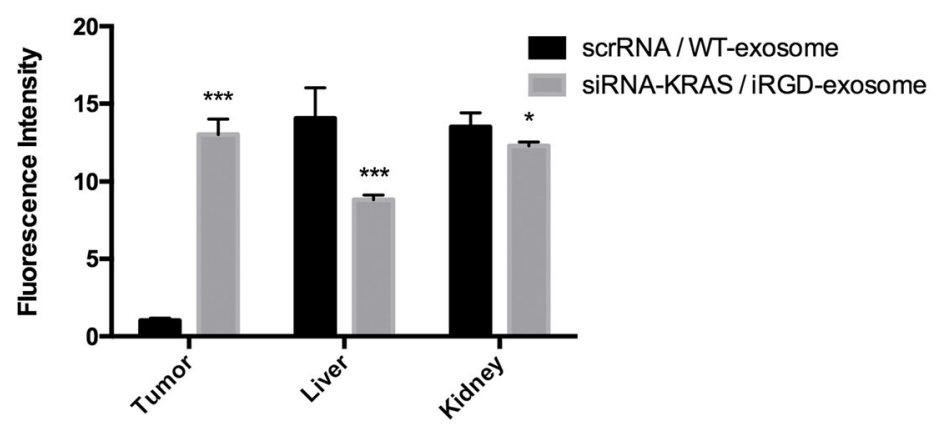

Fig. 3 iRGD-exosomes specially targeted tumor tissues in vivo. (a) The distribution of Dil dye marked exosomes in different organs. (b) The Fluorescence intensity of different organs

fluorescence intensity indicating extensive tumor burden and rapid tumor development was observed in the mice treated with scrRNA/WT-exosomes, tumor growth was significantly reduced in the mice treated with siRNA-KRAS/iRGD-exosomes, and in some cases, tumor growth was completely stopped (Fig. 4a and b). Histopathological examinations of lung lesions revealed highly cellular tumors with considerable cytological atypia in the mice treated with scrambled RNA encapsulated in iRGD-exosomes, whereas the lung tissues obtained from the mice treated with KRAS siRNA encapsulated in iRGD-exosomes showed the features of both cell apoptosis and necrosis (Fig. 4c). In summary, the results reveal the in vivo therapeutic value for the siRNA-KRAS/ iRGD-exosomes to decrease the expression of KRAS in the lung tumors.

\section{Discussion}

KRAS mutation was identified in tumor cells more than 20 years ago, but its clinical importance in cancer therapy just began to be appreciated. Abnormal activation of KRAS is extensively found in many cancer types and is often associated with drug resistance. Scientists have redoubled efforts to disable the mutated KRAS oncogene. Despite much effort spent on searching for inhibitors for activated KRAS, KRAS has proven extraordinarily difficult to target. Now scientists are trying to find another way to target KRAS gene by blocking signals from downstream genes. However, the attempts to identify druggable targets that are linked to KRAS have been challenging, since
KRAS affects many signaling pathways and may function differently in different cancers. Thus, the search for an alternative approach to block KRAS activity or inhibit KRAS expression is highly required.

siRNA is highly dependent on target gene sequence and can theoretically inhibit the expression of any gene with strong specificity, including those traditionally undruggable genes. However, the transformative therapeutic potential of small RNAs has been stymied by a major obstacle-safe and efficient delivery of siRNA in vivo. Although siRNA delivery could theoretically be achieved through injection of synthetic siRNAs, it is enormously difficult to circulate siRNA at constant and high level even though the repeated injections at short intervals are given, as siRNA is rapidly cleared in vivo. Thus, several techniques using viruses or synthetic vehicles (liposomes or polymer nanoparticles) as siRNA agents have been developed. Despite being reasonably efficient in transferring siRNAs in vivo, the procedures for construction and preparation are labor-intensive, and the delivery process may induce severe immune responses and toxicity in host. Recently, direct administration of modified exosomes containing the small RNAs (siRNA and miRNA) of interest unveils a new strategy to deliver and express small RNAs in vivo. Exosomes, as the host's own RNA shuttle service, can be employed to deliver siRNAs and miRNAs to various tissues and organs through the circulation and bio-distribution of themselves. Therefore, it is now possible to silence many 


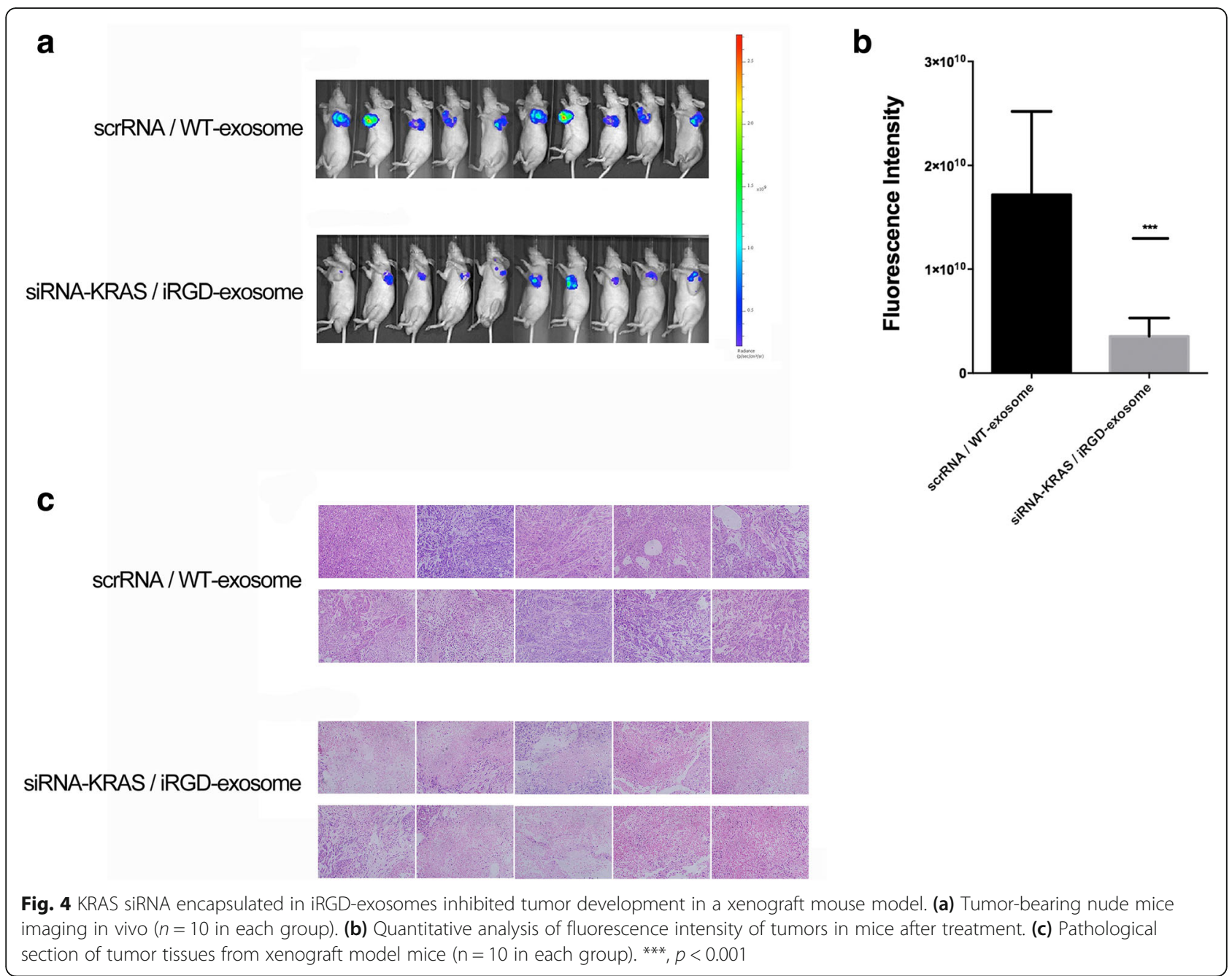

different genes and/or their combinations for their therapeutic activity, even though these genes are located within the tissues and organs (e.g., pancreas and $\mathrm{CD} 4^{+} \mathrm{T}$ cells) that are currently difficult to treat and manipulate by the conventional techniques. This technique is expected to become a promising new siRNA delivery methodology for in vivo gene therapy. In consistent with this concept, we validated in this study that KRAS siRNA encapsulated in iRGD-exosomes could be delivered into tumor cells and efficiently knocked down KRAS expression both in vitro and in vivo. Targeting KRAS with such new strategy may provide a therapeutic option for lung cancer patients with KRAS mutations. As an extension of this finding, the vast majority of mutated genes that are difficult to target by current pharmacological agents will be targetable and druggable in the future.

In conclusion, the present study demonstrates that exosome-based siRNA delivery, which uses iRGD peptide as a guide, is a convenient and efficient mean to induce RNAi in vivo. Employing endogenous exosomes to deliver siRNAs has great significance in theory and application because it can overcome the barriers of delivering siRNA in vivo. Such a technological innovation may reshape the gene therapy field and enable systemic siRNA transfer for treatment of human diseases.

\section{Materials and methods}

\section{Cell culture}

The human embryonic kidney 293 cell line (HEK293T) and adenocarcinomic human alveolar basal epithelial cells cell line A549 were purchased from the Shanghai Institute of Cell Biology, Chinese Academy of Sciences (Shanghai, China). The cells were cultured in high-glucose $(4.5 \mathrm{~g} / \mathrm{L})$ DMEM (Gibco, China) supplemented with 10\% foetal bovine serum (FBS, Gibco, Australia), penicillin and streptomycin in a $5 \% \mathrm{CO}_{2}$, water-saturated atmosphere.

Transfection of HEK293T cells and isolation of exosomes HEK293 cells were cultured in $225-\mathrm{cm}^{2}$ flasks (Corning, China) and were co-transfected with plasmids encoding Lamp2b-iRGD and KRAS siRNA using Lipofectamine 
2000 (Invitrogen) following manufacturer's instructions. The cell culture medium was then collected 2 days after transfection, and the exosomes were harvested from the cultural medium with Exosome Isolation Reagent (Invitrogen). The resulting pellet was then resuspended in PBS.

\section{Western blotting}

Cells were washed with PBS ( $\mathrm{pH}$ 7.4) and then lysed in RIPA Lysis buffer solution (Beyotime, Shanghai, China) supplemented with a protease and phosphatase inhibitor cocktail (Thermo Scientific, Rockford, IL) on ice for 30 min. The tissue samples were frozen solid in liquid nitrogen, powdered and lysed in ice-cold RIPA Lysis buffer containing a protease and phosphatase inhibitor cocktail. The samples were sonicated in an ice bath where necessary. Tissue homogenates and cell lysates were spinned down for $10 \mathrm{~min}\left(12,000 \times \mathrm{g}\right.$ at $\left.4{ }^{\circ} \mathrm{C}\right)$ and the supernatant was collected. The protein levels were normalized by probing the same blots with a GAPDH antibody. KRAS antibody (F234) (SC-30) was supplied by Santa Cruz Biotechnology (Santa Cruz, CA, USA). GAPDH antibody (FL-335) (sc-25,778) was purchased from Santa Cruz Biotechnology (Santa Cruz, CA, USA).

\section{Incubation of cells with exosomes}

Exosomes $(100 \mu \mathrm{g})$ loaded with KRAS siRNA were incubated with A549 cells ( $10^{6}$ cells). After $6 \mathrm{~h}$ incubation, the recipient cells were harvested for total RNA extraction. Subsequently, quantitative RT-PCR analysis of KRAS siRNA and KRAS mRNA and western blot analysis of KRAS protein were performed.

\section{Quantitative RT-PCR assay}

Total RNA was extracted from cells and tissues with TRIzol Reagent (Invitrogen, Carlsbad, CA). Assays used to quantify mature miRNAs were performed using TaqMan miRNA probes (Applied Biosystems, Foster City, CA). One $\mu \mathrm{g}$ of total RNA was reverse-transcribed to cDNA using an AMV reverse transcriptase (TaKaRa, Dalian, China) and Oligo d(T) primer (TaKaRa, Dalian, China) with the following reaction conditions: $16^{\circ} \mathrm{C}$ for $30 \mathrm{~min}, 42^{\circ} \mathrm{C}$ for $30 \mathrm{~min}$, and $85^{\circ} \mathrm{C}$ for $5 \mathrm{~min}$. Real-time PCR was performed using a TaqMan PCR kit and an Applied Biosystems 7300 Sequence Detection System (Applied Biosystems). The reactions were incubated in triplicate in a 96-well optical plate at $95^{\circ} \mathrm{C}$ for $5 \mathrm{~min}$ followed by 40 cycles at $95^{\circ} \mathrm{C}$ for $30 \mathrm{~s}, 65^{\circ} \mathrm{C}$ for 30 s and $72^{\circ} \mathrm{C}$ for $30 \mathrm{~s}$. After the reactions ran to completion, the cycle threshold $\left(\mathrm{C}_{\mathrm{T}}\right)$ values were determined using fixed threshold settings, and the mean $C_{T}$ was determined from the triplicate PCR reactions.

\section{Non-invasive bioluminescence imaging}

Non-invasive bioluminescence imaging was used to assess tumour outgrowth because the in vivo bioluminescence imaging clearly distinguished the tumours from the surrounding tissue even without any contrast agent. Inject $10 \mu \mathrm{l} / \mathrm{g}$ body weight of D-luciferin firefly $(15 \mathrm{mg} / \mathrm{ml}$ in PBS; Caliper Life Sciences Catalog XR-1001) into the mice abdominal cavity $5 \mathrm{~min}$ before anesthetizing the mice by placing it into the gas anesthesia chamber.

\section{NanoSight particle tracking analysis (NTA) of exosomes isolated from HEK293T cell culture media}

The concentration and size of exosomes were detected with the NanoSight NS300 system (Malvern Instruments, Malvern, UK) and configured with scientific CMOS camera with blue $488 \mathrm{~nm}$ laser. Exosomes were diluted in 1 $\mathrm{mL}$ of PBS. The diluted sample was collected and analysed on the NTA software (Version 3.2).

\section{Cell proliferation assay}

A549 cell was incubated with exosomes in plates. After incubation, 5-ethynyl-20-deoxyuridine (EdU) (100 mM) (Cell Light EdU DNA imaging Kit, Guangzhou RiboBio, China) was added and the cells were cultured for $2 \mathrm{~h}$. The cells were then stained according to the following protocol: discard the EdU medium mixture, add $4 \%$ paraformaldehyde to fix cells at room temperature for $30 \mathrm{~min}$, wash with glycine $(2 \mathrm{mg} / \mathrm{mL})$ for $5 \mathrm{~min}$ in a shaker, add $0.2 \%$ Trion X-100 for 10 min, wash with PBS for two times, add click reaction buffer (Tris- $\mathrm{HCl}, \mathrm{pH}$ 8.5, $100 \mathrm{mM} ; \mathrm{CuSO}_{4}, 1 \mathrm{mM}$; Apollo 567 fluorescent azide, $100 \mathrm{mM}$; ascorbic acid, $100 \mathrm{mM}$ ) for $10-30 \mathrm{~min}$ while protecting from light, wash with $0.5 \%$ Triton X-100 for three times, stain with Hoechst $(5 \mathrm{mg} / \mathrm{mL})$ for $30 \mathrm{~min}$ at room temperature, wash with $0.5 \%$ Triton X-100 for five times, and, finally, add $150 \mathrm{ml}$ PBS. Images were taken and analyzed using High Content Imaging Pathway 855 (BD, USA). EdU positive cell was calculated with (EdU add-in cells/Hoechst stained cells) $\times 100 \%$.

\section{Statistical analysis}

All western blot results, histopathological staining and immunohistochemical staining sections shown in this manuscript are representative of at least three independent experiments. Quantitative RT-PCR assays were carried out in triplicate, then, each individual experiment was repeated for statistical accuracies. The data are presented as the mean \pm SEM of at least three independent experiments. Differences were considered at significance of $p<0.05$ using Student's t-test. 


\section{Additional file}

Additional file 1: Figure S1. The construction map of the siRNA-KRAS/ iRGD plasmid. (DOCX $3538 \mathrm{~kb}$ )

\section{Abbreviations}

HEK293T cell: human embryonic kidney 293 T cell; iRGD: internalizing RGD; RNAi: RNA interference; RVG: rabies virus glycoprotein; siRNA: small interfering RNA

\section{Acknowledgements}

Not applicable.

\section{Funding}

This work was supported by the National Natural Science Foundation of China (No. 31871295 and 31741075) and the Natural Science Foundation of Jiangsu Province (No. BE2016737).

\section{Availability of data and materials}

All data generated or analysed during this study are included in this published article.

\section{Authors' contributions}

Xi Chen formulated the hypothesis, commented on this study and edited the manuscript. Yu Zhou and Yuan Yuan performed the experiments and Minghui Liu, Xiuting Hu offered some assistance. Yu Zhou did data analysis and Yiming Quan offered some assistance. All authors reviewed the manuscript. All authors read and approved the final the final manuscript.

\section{Ethics approval and consent to participate}

This study was approved by the Ethics Committee of Nanjing University. All animal care and handling procedures were performed in accordance with the National Institutes of Health's Guide for the Care and Use of Laboratory Animals and were approved by the Institutional Review Board of Nanjing University.

\section{Consent for publication}

Not applicable.

\section{Competing interests}

The authors declare that they have no competing interests.

\section{Publisher's Note}

Springer Nature remains neutral with regard to jurisdictional claims in published maps and institutional affiliations.

Received: 16 October 2018 Accepted: 15 March 2019

Published online: 03 December 2019

\section{References}

1. Rulli $E$, et al. Value of KRAS as prognostic or predictive marker in NSCLC: results from the Tailor trial. Ann Oncol. 2015;26(10):2079-84

2. Shen $\mathrm{H}$, et al. Diagnostic and prognostic value of blood samples for KRAS mutation identification in lung cancer: a meta-analysis. Oncotarget. 2017; 8(22):36812-23.

3. Wennerberg K, Rossman KL, Der CJ. The Ras superfamily at a glance. J Cell Sci. 2005:118(Pt 5):843-6.

4. Adjei AA. Blocking oncogenic Ras signaling for cancer therapy. J Natl Cancer Inst. 2001:93(14):1062-74.

5. Pylayeva-Gupta Y, Grabocka E, Bar-Sagi D. RAS oncogenes: weaving a tumorigenic web. Nat Rev Cancer. 2011;11(11):761-74.

6. Lemstrova R, et al. Dysregulation of KRAS signaling in pancreatic cancer is not associated with KRAS mutations and outcome. Oncol Lett. 2017;14(5): 5980-8.

7. Kim HS, et al. The impact of KRAS mutations on prognosis in surgically resected colorectal cancer patients with liver and lung metastases: a retrospective analysis. BMC Cancer. 2016;16:120.

8. Karachaliou N, et al. KRAS mutations in lung cancer. Clin Lung Cancer. 2013; 14(3):205-14.
9. Baulcombe D. Overview of RNA interference and related processes: John Wiley \& Sons; 2003, Inc. Unit 26.1.

10. Thery C, Zitvogel L, Amigorena S. Exosomes: composition, biogenesis and function. Nat Rev Immunol. 2002;2(8):569-79.

11. Mathivanan S, Ji H, Simpson RJ. Exosomes: extracellular organelles important in intercellular communication. J Proteome. 2010;73(10):1907-20.

12. Simons M, Raposo G. Exosomes--vesicular carriers for intercellular communication. Curr Opin Cell Biol. 2009;21(4):575-81.

13. Simeoli $R$, et al. Exosomal cargo including microRNA regulates sensory neuron to macrophage communication after nerve trauma. Nat Commun. 2017;8(1):1778.

14. Pitt JM, Kroemer G, Zitvogel L. Extracellular vesicles: masters of intercellular communication and potential clinical interventions. J Clin Invest. 2016; 126(4):1139-43.

15. Zhou Y, et al. Exosome-mediated small RNA delivery for gene therapy. Wiley Interdiscip Rev RNA. 2016

16. Liu Y, et al. Targeted exosome-mediated delivery of opioid receptor mu siRNA for the treatment of morphine relapse. Sci Rep. 2015;5:17543.

17. Alvarez-Erviti $L$, et al. Delivery of siRNA to the mouse brain by systemic injection of targeted exosomes. Nat Biotechnol. 2011;29(4):341-5.

18. Gao Y, et al. RVG-peptide-linked trimethylated chitosan for delivery of siRNA to the brain. Biomacromolecules. 2014;15(3):1010-8.

\section{Ready to submit your research? Choose BMC and benefit from:}

- fast, convenient online submission

- thorough peer review by experienced researchers in your field

- rapid publication on acceptance

- support for research data, including large and complex data types

- gold Open Access which fosters wider collaboration and increased citations

- maximum visibility for your research: over $100 \mathrm{M}$ website views per year

At $\mathrm{BMC}$, research is always in progress.

Learn more biomedcentral.com/submissions 